\title{
Augmented models for improving vision control of a mobile robot
}

\author{
Andersen, Gert Lysgaard; Christensen, Anders C.; Ravn, Ole
}

Published in:

Proceedings of the Third IEEE Conference on Control Applications

Link to article, DOI:

10.1109/CCA.1994.381270

Publication date:

1994

Document Version

Publisher's PDF, also known as Version of record

Link back to DTU Orbit

Citation (APA):

Andersen, G. L., Christensen, A. C., \& Ravn, O. (1994). Augmented models for improving vision control of a mobile robot. In Proceedings of the Third IEEE Conference on Control Applications (Vol. Volume 1, pp. 53-58). IEEE. https://doi.org/10.1109/CCA.1994.381270

\section{General rights}

Copyright and moral rights for the publications made accessible in the public portal are retained by the authors and/or other copyright owners and it is a condition of accessing publications that users recognise and abide by the legal requirements associated with these rights.

- Users may download and print one copy of any publication from the public portal for the purpose of private study or research.

- You may not further distribute the material or use it for any profit-making activity or commercial gain

- You may freely distribute the URL identifying the publication in the public portal 


\title{
AUGMENTED MODELS FOR IMPROVING VISION CONTROL OF A MOBILE ROBOT
}

\author{
Gert L. Andersen, Anders C. Christensen, Ole Ravn \\ Institute of Automatic Control Systems. Bldg. 326 \\ The Technical University of Denmark \\ DK-2800 Lyngby, Denmark
}

\begin{abstract}
This paper describes the modelling phases for the design of a path tracking vision controller for a three wheeled mobile robot. It is shown, that by including the dynamic characteristics of vision and encoder sensors and implementing the total system in one multivariable control loop, one obtains good performance even when using standard low cost equipment and a comparatively low sampling rate. The plant model is a compound of kinematic, dyna$\mathrm{mic}$ and sensor submodels, all integrated into a discrete state space representation. An intelligent strategy is applied for the vision sensor, and includes the start up. normal operation, exception handling and shut down phases. Laboratory experiments show the validity of the approach using a standard Kalman Filter / LQR control design.
\end{abstract}

\section{Introduction}

One of the topics within robotic research is computer vision. The use of vision is motivated by the continuing need to increase the flexibility and scope of applications of robotic systems.

The advances in vision technology, i.e. sensors and image processing equipment, has made it possible to introduce vision sensors into real time control systems. Vision is a multi-purpose sensor as different algorithms can be used during different modes of operation. The functionality of the sensor can be reconfigured on-line to adapt to different modes of operation [1].

It is straightforward, when designing the manoeuvering control for a mobile robot. to use a layered architecture of wheel servo / autopilot / navigation pilot, where wheel velocity servoes are implemented as inner loops, and the autopilot uses a kinematic model only. The problem in this approach is that upper levels demand lower levels to be dynamically negligible: the higher maximal speed of the robot required, the higher demands for the speed and

$0-7803-1872-2 / 94 / \$ 4.00$ @ 1994 IEEE accuracy of the equipment. This goes for the bandwidth of the wheel servoes, precision of the encoders, image sensiñ and processing speed. For fairly normal speeds these bandwidth requirements exceed microprocessors of standard price levels.

We design a controller that can keep a mobile robot following a predefined track using vision feedback. In order to minimize cost and complexity of equipment, the design is based on a one-level modelling of the robot and the sensors involved.

The outline of the paper is as follows. First we describe the mobile robot and the special mode used for navigation using vision feedback. Then we derive a discrete-time model, which is a compound model, combining three different submodels: a kinematic, a dynamic and a sensor model. We apply standard methods to carry out the observer and controller design. Finally, we validate the closed loop performance in laboratory experiments.

\section{The Mobile Robot}

During the last years, a 2 DOF mobile robot has been constructed at the Institute of Automatic Control Systems, see figure 1.

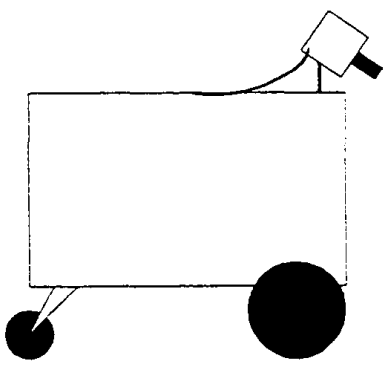

Figur 1. Sketch of the mobile robot at IACS.

The mobile robot serves as a test bed for the comparisons of different algorithms for control of intelligent vehicles on equal terms, without letting the implementation differences play a major role. The robot is built using standard components 
instead of buying a ready made vehicle as this gives more control of the degree of flexibility and versatility, [2].

The robot is moveded and steered by two independently driven front wheels and at the rear end there is a castor wheel for stabilty. For the purpose of controlling the basic motions, the robot is equipped with digital encoders on each of the motor shafts, and a CCD camera is placed in front of the robot in a pan and tilt mechanism. The robots main dimensions are: $70 \mathrm{~cm}$ long. $50 \mathrm{~cm}$ wide, $55 \mathrm{~cm}$ high. The total weight is $70 \mathrm{~kg}$.

The position of the camera. relative to the center point of the vehicle, is defined by the height above floor level, $h$, and the displacement ahead of the center line, $l$. The camera orientation is defined by the tilt angle, $\theta_{r}$, and the pan angle, $\theta_{p}$, figure 2 .

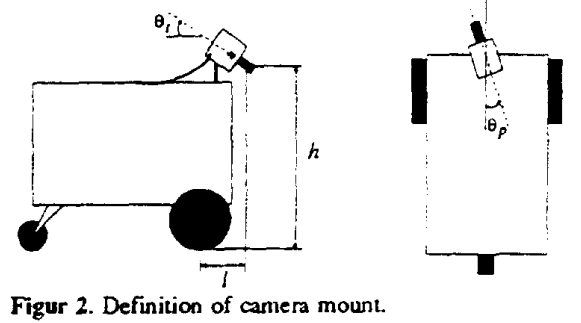

The Highway Mode

In this paper, the mobile robot is operated in highway mode, e.g. it follows a black tape track on the floor using a vision sensor, a vision algorithm, the encoder sensors and a control algorithm.

Stated in automation terms, i.e. start up, normal operation, exception handling, shut down, the structure of the the vision algorithm can be explained as follows:

During start up, the robot is at rest while the overall picture is searched for the track.

During normal operation, the robot is moving at maximum velocity. The vision algorithm scans for the track in a line in the bottom of the sensor picture and another in the top, figure 3.

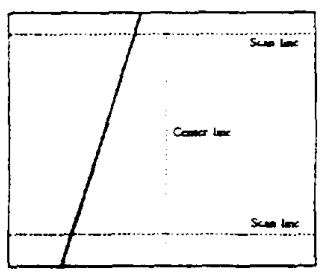

Figur 3. The view of the camera during highway mode. Two horizontal scan-lines and the center line are indicated.

For stopping at intersections and decreasing speed for possible turns, the top line is scanned for intersecting tracks as well.
If the track is changing too rapidly, if the tracking becomes poor, or if the track is lost in the sensor picture, then, the vision algorithm uses its exception handling routine. In this particular case, the exception handling routine decreases the velocity of the robot to a predefined level using constant deceleration. When the path tracking is reestablished and the track seems straight, the velocity is increased to maximum again. If the track is lost and not found again. the robot is stopped (shut down).

In highway mode, the vision algorithm outputs the lateral position error, found as the distance from the track to the center line of the sensor picture. A picture is scanned at every $40 \mathrm{~ms}$ and saved in the computer RAM, then a match filter is used in order to find the track. The lateral position error is found and intersecting tracks are sought for.

\section{Modelling}

The following models are needed in order to be able to use standard methods for the controller design:

A kinematic model which expresses the relationship between the lateral position error and the angular velocity of the robot.

A dynamic model which expresses the relationship between linear and angular velocities of the robot and the input voltages to the DC steering motors. A sensor model which expresses the relationship between the physical quantities being measured and the measurements available from the sensor.

Finally, a discrete-time model combining the kinematic, the dynamic and the sensor models.

\section{Kinematic Model}

When the kinematic model is derived, i.e. finding the connection between the hateral position error, $x_{e}$, and the angular velocity of the robot, $\omega$, the dynamics are neglected. Further it is assumed that the robot moves at a constant speed, $V$. Following figure 4 , some geometric considerations result in the set of equations (1), (2) and (3) :

$$
\begin{gathered}
x_{r}=L \tan (\theta)+\frac{x_{0}}{\cos (\theta)} \\
\dot{x}_{0}=V \sin (\theta) \\
\theta=\omega=\frac{r}{b}\left(\omega_{r}-\omega_{l}\right)
\end{gathered}
$$

where $\omega_{r}$ and $\omega_{1}$ are angular velocities of right and left wheel, and $r$ and $b$ are wheel-radius and base, respectively. $L$ is the distance between the point of rotation and the point where the lateral position error is measured, $x_{0}$ is the lateral displacement of the robot and $\theta$ its orientation relative to the track. 


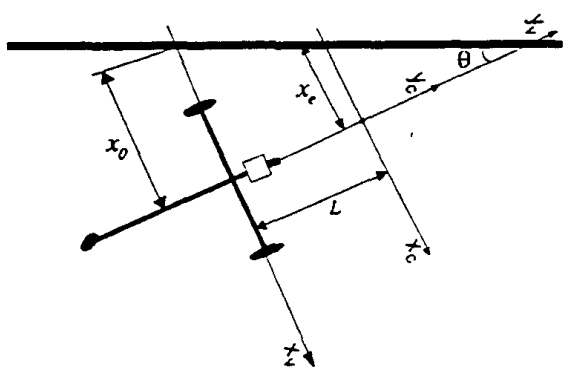

Figur 4. The robot placed close to the track.

Assuming small deviations from $\theta=0^{\circ}$, the following matrices in a standard state space description are obtained:

$$
\begin{array}{cc}
A_{k}=\left[\begin{array}{ll}
0 & 0 \\
V & 0
\end{array}\right] & B_{k}=\left[\begin{array}{cc}
\frac{r}{b} & \frac{-r}{b} \\
0 & 0
\end{array}\right] \\
C_{k}=\left[\begin{array}{ll}
L & 1
\end{array}\right] . & D_{k}=[0]
\end{array}
$$

where the state and input vectors are:

$$
x^{T}=\left[\begin{array}{ll}
\theta & x_{0}
\end{array}\right], \quad u^{T}=\left[\begin{array}{ll}
\omega, & \omega_{t}
\end{array}\right]
$$

\section{Dynamic Model}

The dynamic model is derived from the theory of rigid systems. A rigid system is defined as a system in which the distance between its parts do not change. Planar motions for rigid systems are described as the sum of translation and rotation. In the following superscripts $w$ and $m$ are referring to wheel and motor, respectively.

The dynamic model is derived in a coordinate system fixed in the vehicle body, see figure 5 .

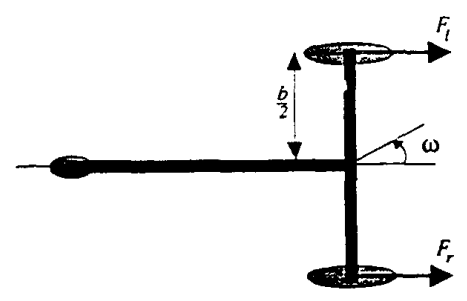

Figur 5. Sketch of a three wheeled robot.

Using Newtons second law around the robots center point the following equations may be derived:

$$
\begin{gathered}
M \dot{v}=F_{r}+F_{l} \\
J \omega=\frac{b}{2} F_{r}-\frac{b}{2} F_{l}
\end{gathered}
$$

where $v$ and $\omega$ denote translational and angular velocities, $I$ the moment of inertia, and $M$ the total mass of the robot. $F_{r}$ and $F_{r}$ are right and left wheel force, respectively.

Solving (7) and (8), the following expressions for the force on the wheels are obtained:

$$
\begin{aligned}
& F_{r}=\frac{M}{2} \dot{v}+\frac{I}{b} \dot{ } \\
& F_{1}=\frac{M}{2} \dot{v}-\frac{I}{b} \omega
\end{aligned}
$$

We have the following expressions for the connection between the translational and angular velocities of the center point $v$ and $\omega$ and the wheel velocities

$$
\begin{aligned}
& v=\frac{v_{r}{ }^{\omega}+v_{i}{ }{ }}{2} \\
& \omega=\frac{v_{i}{ }^{\omega}-v_{l}{ }^{\omega}}{b}
\end{aligned}
$$

Similar expressions are obtainable for the linear and angular acceleration, by simple differentiation.

Likewise, we have the relationships (13) and (14) between angular velocity of the wheel axle $\omega^{\omega}$ and wheel velocity $v^{w}$, and torque $\tau$ on the motor shaft and wheel force.

$$
\begin{aligned}
v^{w} & =r \omega^{w} \\
\tau & =\frac{r}{N} F
\end{aligned}
$$

where $r$ is the wheel radius and $N$, is the gear ratio between the motor and wheel axle.

Inserting the expressions for $v, \omega, v^{w}$, and $\tau$ in the wheel force equations (9) and (10), one obtains

$$
\begin{aligned}
\tau_{r}^{m} & =\frac{r^{2}}{N}\left(\frac{M}{4}+\frac{I}{b^{2}}\right) \omega_{r}^{\omega}+\frac{r^{2}}{N}\left(\frac{M}{4}-\frac{I}{b^{2}}\right) \omega_{l}^{\omega} \\
& =k_{r} \omega_{r}^{\omega}+k_{l r} \omega_{l}^{\omega} \\
\tau_{l}^{m} & =\frac{r^{2}}{N}\left(\frac{M}{4}-\frac{I}{b^{2}}\right) \omega_{r}^{\omega}+\frac{r^{2}}{N}\left(\frac{M}{4}+\frac{I}{b^{2}}\right) \omega_{l}^{\omega} \\
& =k_{r l} \omega_{r}^{\omega}+k_{l} \omega_{l}^{\omega}
\end{aligned}
$$

expressing the relation between the wheel angular accelerations and the torque on the motor shafts.

It is now possible to obtain a model, expressing the dynamic behaviour from the input to the DC motors to the wheel velocities, that includes the coupling between the two driven wheels.

Figur 6 shows a block diagram for the two DC motors including the cross couplings $k_{i r}$ and $k_{r l}\left(k_{e}\right.$, $k_{i}, R$ and $J$ are the motor constants). 


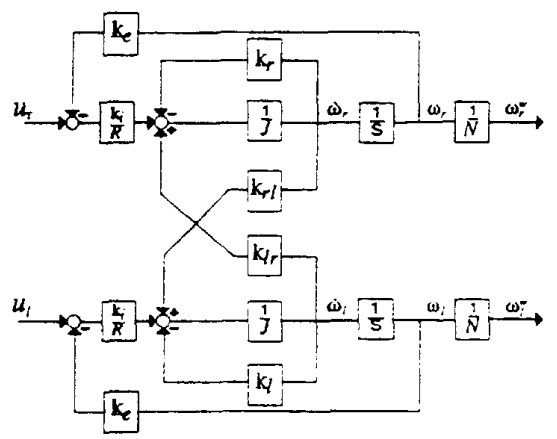

Figur 6. Block diagram representing the robot dynamics. Subscripts $r$ and $l$ are omitted for the motor constants.

Choosing as state vector the angular velocities of the wheel axles and as input vector the ancre voltage to the $\mathrm{DC}$ - motors

$$
x^{T}=\left[\begin{array}{ll}
\omega_{r}^{\omega} & \omega_{i}^{\omega}
\end{array}\right], \quad u^{T}=\left[\begin{array}{ll}
u_{r} & u_{l}
\end{array}\right]
$$

the matrices in a standard linear state space model is given by equations (18) - (20) below.

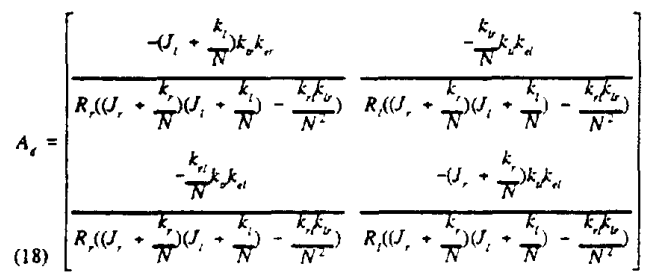

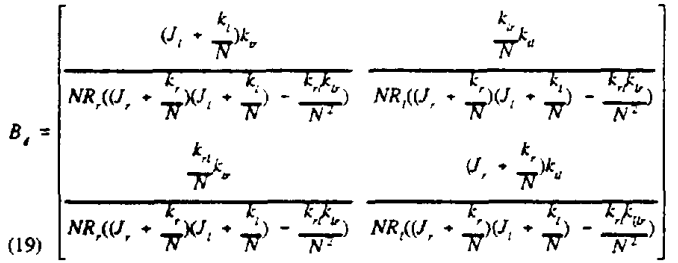

$$
C_{d}=\left[\begin{array}{ll}
r / 2 & r / 2
\end{array}\right] \quad D_{d}=\left[\begin{array}{ll}
0 & 0
\end{array}\right]
$$

Combining the kinematic and dynamic models (4) (6) and (17) - (20), one obtains the following augmented continuous-time model.

$$
\begin{aligned}
& \boldsymbol{u}^{T}=\left[\begin{array}{ll}
u_{r} & u_{l}
\end{array}\right] \\
& x^{T}=\left[\begin{array}{llll}
\omega_{r} & \omega_{1} & \theta & x_{0}
\end{array}\right] \\
& y^{T}=\left[\begin{array}{ll}
V & x
\end{array}\right] \\
& A=\left[\begin{array}{cc}
A_{d} & 0 \\
B_{k} & A_{k}
\end{array}\right] \quad B=\left[\begin{array}{l}
B_{d} \\
0
\end{array}\right] \\
& C=\left[\begin{array}{l}
C_{c} \\
C_{c}
\end{array}\right]=\left[\begin{array}{ll}
C_{d} & 0 \\
0 & C_{k}
\end{array}\right] \quad D=\left[\begin{array}{l}
D_{c} \\
D_{c}
\end{array}\right]=\left[\begin{array}{l}
0 \\
0
\end{array}\right]
\end{aligned}
$$

\section{Sensor Model}

In order to measure velocity and lateral position error, the robot is equipped with two different types of digital sensors. The furst type is a standard surveillance camera with a frame grabber and an onboard computer. The second type is a set of digital encoders placed on each of the motor shafts.

The Vision Sensor. To perform mobile robot navigation from a robot mounted camera, it is important to be able to interpret the locations of features observed in the image. This involves determining both the intrinsic camera parameters and the positioning of the camera mount with respect to the robot.

A simple linear model for the relationship between distances measured in world coordinates. $(x, y)$, and the result in camera coordinates, $(\Delta x, \Delta y)$, is:

$$
\begin{aligned}
& \frac{x}{L}=\frac{\Delta x}{f_{x}} \\
& \frac{y}{L}=\frac{\Delta y}{f_{y}}
\end{aligned}
$$

This model is described by 4 intrinsic parameters: the focal lengths in vertical and horizontal direction, $\left(f_{x}, f_{y}\right)$, and the position of the center point in the picture. $L$ is the distance between the object and picture plane, i.e. the distance between the camera and the object. The intrinsic parameters are fixed for a certain adjustment of the camera (zoom and focus) and are found by a standard calibration method. The variation of the intrinsic parameters, due to distortion, is less than 5\% [3] and can thus neglected.

Due to the sensing, preprocessing and segmentation processes, the camera sensor introduces a time delay into the control loop. In the present implementation, the delay is constant and equals $\Gamma_{d}=35 \mathrm{~ms}$, as opposed to a sampling period of $T=80 \mathrm{~ms}$.

The presence of the camera results in an augmentation of the plant model. Assuming a continuous, time invariant system $[A, B, C]$ with a zero-order hold at the input, and a time delay of a fraction $0<d<1$ of the sampling period $T$, one obtains

$$
\begin{gathered}
x(k+1)=A^{*} x(k)+B^{*} u(k) \\
y(k)=C_{c i}^{\cdot} x(k)+C_{c}^{*} u(k-1)
\end{gathered}
$$

where $A^{*}$, and $B^{*}$ denotes the discrete representations of the continuous system and $C_{c l}$ and $C_{c 2}$ are

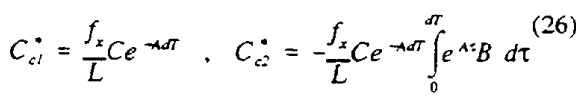

with $f_{x}$ and $L$ defined as in (24). 
A standard state space form is obtained by representing the delayed control signal as a state:

$$
\begin{gathered}
{\left[\begin{array}{c}
x(k+1) \\
x_{\mathrm{r}}(k+1)
\end{array}\right]=\left[\begin{array}{cc}
A^{*} & 0 \\
0 & 0
\end{array}\right]\left[\begin{array}{l}
x(k) \\
x_{\mathrm{r}}(k)
\end{array}\right]+\left[\begin{array}{l}
B^{\cdot} \\
I
\end{array}\right] u(k)} \\
y(k)=\left[\begin{array}{ll}
C_{c l}^{*} & C_{c \cdot}^{\cdot}
\end{array}\right]\left[\begin{array}{l}
x(k) \\
x_{\mathrm{r}}(k)
\end{array}\right]
\end{gathered}
$$

The Digital Encoder. The mobile robot has digital encoders placed directly on each of the motor shafts. The encoders work as reset integrators, i.e they are read at each sampling instance and then reset to zero. This measurement expresses the distance driven during one sampling period, and can be interpreted as an average velocity.

A reset integrator is represented in the following way:

$$
z(k)=\int_{0}^{\tau} y(\tau) d \tau
$$

Here $y(\tau), k<\tau<k+1$ can be expressed as

$$
y(\tau)=C e^{\wedge(\tau)} x(k)+C \int_{0}^{\tau} e^{\wedge(\tau-s)} B d s u(k)
$$

assuming that the system $[A, B, C]$ is continuous, linear, time invariant, and includes a zero-order hold at the input.

Combining (28) and (29), and assuming a sampling period $T$ results in

$$
z(k)=A_{e}^{*} x(k)+B_{e}^{*} u(k)
$$

where $A_{e}^{*}$ and $B_{e}^{*}$ are given by:

$$
\begin{gathered}
A_{c}^{*}=\int_{0}^{T} C e^{A \tau} d \tau \\
B_{c}^{*}=\int_{0}^{T} \int_{0}^{\tau} C e^{A(\tau-s)} B d s d \tau
\end{gathered}
$$

A digital encoder model introduces a new state into the resulting discrete-time system. The augmented system can be expressed, assuming $D^{*}=0$, as

$$
\begin{gathered}
{\left[\begin{array}{l}
x(k+1) \\
z(k+1)
\end{array}\right]=\left[\begin{array}{ll}
A^{*} & 0 \\
A_{e}^{*} & 0
\end{array}\right]\left[\begin{array}{l}
x(k) \\
z(k)
\end{array}\right]+\left[\begin{array}{l}
B^{\cdot} \\
B_{;}^{*}
\end{array}\right] u(k)} \\
y(k)=\left[\begin{array}{ll}
0 & C_{\bullet}^{*}
\end{array}\right]\left[\begin{array}{l}
x(k) \\
z(k)
\end{array}\right]
\end{gathered}
$$

where $\left[A^{*}, B^{*}, D^{*}\right]$ is the discrete representation of the continuous-time system.
Referring to the continuous-time plant equations above, we measure the translational velocity of the robot by applying the encoders to right and left shaft, respectively ( $k_{\text {enc }}$ is the encoder gain):

$$
\left[\begin{array}{c}
z_{r}(k) \\
z_{l}(k)
\end{array}\right]=N k_{\text {enc }} \int_{0}^{\tau}\left[\begin{array}{l}
\omega_{r}(\tau) \\
\omega_{l}(\tau)
\end{array}\right] d \tau
$$

thus obtaining

$$
V(k)=\left[\begin{array}{ll}
\frac{r}{2} & \frac{r}{2}
\end{array}\right]\left[\begin{array}{l}
z_{r}(k) \\
z_{r}(k)
\end{array}\right]
$$

hence,

$$
C_{e}^{*}=\left[\begin{array}{lll}
0 & \frac{r}{2} & \frac{r}{2}
\end{array}\right]
$$

\section{Resulting Discrete Model}

Combining the different models described above and the sampling process results in a linear time invariant discrete state space model of $8^{\text {th }}$ order.

$$
\begin{gathered}
u_{a}^{T}=\left[\begin{array}{ll}
u_{,} & u_{l}
\end{array}\right] \\
x_{a}{ }^{T}=\left[\begin{array}{lll}
x & x_{\mathrm{r}} & z
\end{array}\right] \\
A_{a}^{*}=\left[\begin{array}{ccc}
A^{*} & 0 & 0 \\
0 & 0 & 0 \\
A_{c}^{*} & 0 & 0
\end{array}\right] \quad B_{a}^{*}=\left[\begin{array}{c}
B^{*} \\
I \\
B_{\dot{c}}^{*}
\end{array}\right] \\
C_{a}^{*}=\left[\begin{array}{ccc}
C_{c}^{*} & C_{c 2}^{*} & 0 \\
0 & 0 & C_{e}^{*}
\end{array}\right] \quad D_{a}^{*}=\left[\begin{array}{l}
0 \\
0
\end{array}\right]
\end{gathered}
$$

The matrix exponential integrals in (26), (31) and (32), are solved using a result from [4].

To ensure that the velocity of the robot has zero steady state error, the system is augmented with an integral state, resulting in a $9^{\text {th }}$ order model.

The system is completely observable and controlable. The A matrix is singular due to the extra states introduced by the camera and encoder models.

\section{Design of vision controller}

This section summarizes briefly the controller design. The controller is based on state feedback and is designed using standard LQ techniques.

\section{Kalman Filter}

For state reconstruction an optimal Kalman filter has been designed. The Kalman filter is implemented in a way that uses the latest measurements for state update, [5]. 
The state and measurement noise is due to the quantization in the DA converter, the camera and encoders, respectively. Quantization noise can be approximated by uncorrelated discrete-time white noise with variance $h^{2} / 12, h$ being the smallest representable unit, [6]. The resolution of the DA converer, camera, and encoders equals $h_{\mathrm{DA}}=5.0 \mathrm{mV}$, $h_{\mathrm{cos}}=1.0 \mathrm{~mm}, h_{\mathrm{exc}}=0.7 \mathrm{~mm}$, respectively.

\section{Controller}

The controller gains are found using LQR techniques. The states and inputs (21) are weighted using diagonal weight matrices in continuous time. These are then transformed to equivalent discrete weight matrices. The discrete-time weight matrices include cross product terms that penalize the product of the states and inputs. [7].

\section{Performance}

To validate the performance of the closed loop control system and the applied sensor strategy the robot is commanded to follow a tape track, having two lateral shifts. figure 7 . The slope of the shifts equals -0.6 and 0.35 respectively.

During the experiment the vision algorithm varies the velocity of the vehicle between $0.3-0.8 \mathrm{~m} / \mathrm{s}$ with the accelation fixed to $0.5 \mathrm{~m} / \mathrm{s}^{2}$.

Figure 7 shows a reconstruction of the robot motion based on physical encoder measurements, using a simple robot simulator. Figure 8 shows the path tracking, i.e. the lateral position error measured by the camera. Figure 9 and 10 show's the translational and rotational velocity of the robot.

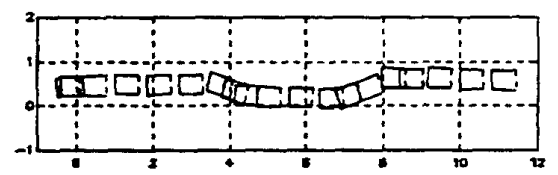

Figur 7. Robot movements in a $(x[m] . y[m])$ frame.

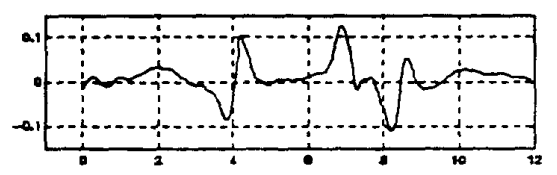

Figur 8. Lateral position error. Units: $[\mathrm{m}]$ versus $[\mathrm{m}]$.

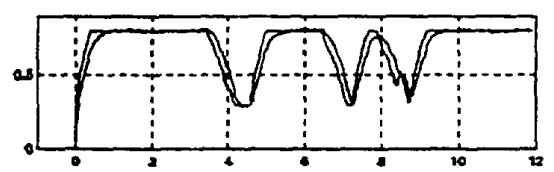

Figur 9. Translational velocity. Units: $[\mathrm{m}]$ versus $[\mathrm{m} / \mathrm{sec}]$.

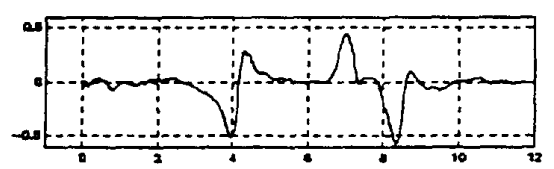

Figur 10. Angular velocity. Units: [m] versus [rad/sec].
The system keeps the tracking error within $\pm 0.02 \mathrm{~m}$, except when the reference is changing. As seen from figures 7 and 10 the robot moves smoothly, showing no tendency to oscillate, keeping in mind that the robot drives between $6.4 \mathrm{~cm}$ and $2.4 \mathrm{~cm}$ during each sample.

\section{Conclusion}

The work described in this paper shows how well known standard modelling and design techniques can be combined to solve the path tracking problem using vision feedback. We have obtained a closed loop control system that performs well, despite of low cost equipment, (standard surveillance camera. digital encoders, low sampling rate).

The keys to succes has been a proper modeling of the dynamical system including the applied sensors and the application of an intelligent strategy for the vision sensor.

\section{Acknowledgements}

The basic vision algorithms are implemented by Allan Holmegaard and described in [8].

\section{References}

[1] Andersen, N.A., Ravn, O., Sørensen, A.T., "Real-time vision based control of servomechanical systems", Proc. of the Second International Symposium on Experimental Robotics, France, 1991.

[2] Ravn,O., Andersen, N.A., "A test bed for experiments with intelligent vehicles", Proc. of Ist IFAC international Workshop on Intelligent Autonomous Vehicles, Hampshire, United Kingdom. 1992.

[3] Ravn, O., Andersen, N.A., Sørensen, A.T., "Auto-calibration in automation systems using vision", Proc. of the Third International Symposium on Experimental Robotics, Japan, 1993.

[4] Loan, C.F.V., "Computing integrals involving the matrix exponential", IEEE Transactions on Automatic Control, vol AC-23, no. 3, 1978.

[5] Kwakernaak, H., Sivan, R., Linear optimal control systems, Wiley-Interscience, 1972.

[6) Levin, M.L., Datamatbaserede reguleringssytermer, Servolaboratoriet, DiH. (in danish), 1982.

[7] Franklin, G.F., Powell, J.D., Workman, M.L. Digital control of dynamic systems, Second Edition. Addison-Wesley Publishing Compagny, 1990.

[8] Holmegaard, A.Z., Robust regulering of $A G V$, Master Project, Servolaboratoriet, DTH, 1992. 